Revue d'histoire de l'Amérique française

BRS REVUE D.HISTOIRE DE L'AMÉRIQUE FRANÇAISE

\title{
Les échanges scientifiques entre Roland-Michel Barrin de La Galissonière et les chercheurs contemporains
}

\section{Roland Lamontagne}

Volume 14, numéro 1, juin 1960

URI : https://id.erudit.org/iderudit/302028ar

DOI : https://doi.org/10.7202/302028ar

Aller au sommaire du numéro

Éditeur(s)

Institut d'histoire de l'Amérique française

ISSN

0035-2357 (imprimé)

1492-1383 (numérique)

Découvrir la revue

Citer cet article

Lamontagne, R. (1960). Les échanges scientifiques entre Roland-Michel Barrin de La Galissonière et les chercheurs contemporains. Revue d'histoire de l'Amérique française, 14(1), 25-33. https://doi.org/10.7202/302028ar d'utilisation que vous pouvez consulter en ligne. 


\section{LES ÉCHANGES SCIENTIFIQUES ENTRE ROLAND-MICHEL BARRIN DE LA GALISSONIĖRE ET LES CHERCHEURS CONTEMPORAINS *}

Le ministre de la Marine, Louis Phélypeaux de Pontchartrain, avait proposé à Louis XIV, en décembre 1699, d'envoyer à l'étranger des personnes capables de faire des observations en histoire naturelle et en géographie. Louis XIV avait approuvé le président de l'Académie Royale des Sciences, l'abbé Bignon, qui avait choisi le botaniste Joseph Pitton de Tournefort. ${ }^{1}$ L'intendant général des galères à Marseille, Michel Bégon, organisera sur ordre du roi des expéditions scientifiques aux Antilles dont il confiera la direction au Père Charles Plumier. ${ }^{2}$

Il arrive que les botanistes expriment leur reconnaissance en désignant une plante d'après le nom d'un personnage. Le $\mathrm{P}$.

* Communication présentée lors de la journée d'étude de l'Institut d'Histoire de l'Amérique française, le 23 avril 1960.

Je poursuis une étude d'ensemble sur l'œuvre colonisatrice de La Galissonière. $\mathrm{Au}$ cours de mes recherches, je ne pouvais pas ignorer la participation de ce gouverneur à l'activité scientifique de son époque.

La documentation a été facilitée par l'empressement avec lequel ont répondu à mes demandes de microfilms ou de renseignements $\mathbf{M}^{11 \mathrm{e}} \mathrm{M}$. Chabrier, chef du service photographique de la Bibliothèque Nationale de Paris, le Révérend Père Paul Desjardins, S.J., archiviste du Collège Sainte-Marie, M. Jacques Rousseau, alors directeur du Musée National à Ottawa, M. F. Dousset, adjoint du directeur général des Archives de France, M. David C. Mearns, conservateur du département des manuscrits de la Bibliothèque du Congrès, M Mlle Anne-Marie Pfister, bibliothécaire aux manuscrits de la Bibliothèque publique et universitaire de Genève. M. Yves Laissus, archiviste-paléographe du Muséum national d'histoire naturelle, a eu l'obligeance de me communiquer une copie exactement faite d'une lettre de JeanFrançois Gaulthier à Jean-Etienne Guettard.

Il m'est agréable d'exprimer mes remerciements pour cette aide qui m'a été apportée avec bienveillance. $R$. L.

1 Pierre Guiral, «Tournefort et son voyage au Levant», Tournefort. Les Grands Naturalistes Français, collection dirigée par Roger Heim (Paris, 1957), 77.

2 J.-P. Coste, « La Botanique à Aix », Ibid., 47. 
Plumier a dédié le bégonia ${ }^{3}$ à Michel Bégon, grand-père de Roland-Michel Barrin de La Galissonière. ${ }^{4}$

Dans un chapitre intitulé : «Le siècle de la curiosité », Charles Morazé a étudié l'action de la société sur la science au XVIII ${ }^{e}$ siècle. ${ }^{5}$ Les membres de l'aristocratie ont fait sortir les préoccupations scientifiques des limites étroites des académies et des sociétés savantes; chacun d'eux désirait posséder un cabinet de physique et d'histoire naturelle. Au XVIII ${ }^{\mathrm{e}}$ siècle, il y avait pour les sciences de la nature un "véritable engouement ${ }^{6}$ qui atteignait les différentes classes sociales. ${ }^{7}$

Auguste Pageot ${ }^{8}$ a présenté Roland-Michel Barrin de La Galissonière sous l'aspect d'un grand seigneur, collectionneur de curiosités naturelles. L'intérêt que La Galissonière a porté aux sciences n'était pas restreint à la récolte d'échantillons d'histoire naturelle. Il s'est manifesté dans la correspondance échangée avec quelques-uns des savants français les plus réputés de la première moitié du XVIII ${ }^{\mathrm{e}}$ siècle. En 1752 , paraissait un opuscule $^{9}$ attribué à Duhamel du Monceau et à Roland-Michel Barrin de La Galissonière. ${ }^{10}$ Celui-ci a annoté le manuscrit de la Flore de Jean-François Gaulthier, ${ }^{11}$ correspondant de Duhamel du Monceau.

3 Ibid., 50.

4 Yvonne Bezard, Fonctionnaires maritimes et coloniaux sous Louis $X I V$ : les Bégon (Paris, 1932).

5 René Taton, éd., La Science moderne. Collection d'histoire générale des sciences (Paris, 1958), 2: 425-431.

${ }^{6}$ L'expression est de Roland Mousnier. Le chapitre intitulé: «L'esprit du siècle » résume les conditions de travail intellectuel au XVIII' siècle; une section de cette étude porte sur les effets de l'engouement du public. Le XVIIIe siècle, révolution intellectuelle, technique et politique, 1715-1815. Histoire générale des civilisations publiée sous la direction de Maurice Crouzet ( 7 vol., 1953-1957), 5: 10-15.

7 D. Roller et D. H. D. Roller, The Development of the Concept of Electric Charge (Harvard University Press, 1954), 49.

${ }^{8}$ Les Amiraux Barrin de La Galissonière et Louis Turpin (Paris, 1950).

9 Avis pour le transport par mer des arbres, des plantes vivaces, des semences, des animaux et de différents autres morceaux d'histoire naturelle (s. 1.).

10 Catalogue général des livres imprimés de la Bibliothèque Nationale (Paris, 1925), tome LXXXVI, 125-126.

11 Archives de la Province de Québec. Il est à souhaiter que soit entreprise la publication d'une édition annotée de la Flore de Jean-François Gaulthier. L'un de nos savants, M. Jacques Rousseau, y travaille, croyonsnous. Espérons qu'il aura le temps de mener cet ouvrage à terme. 
Le $1^{\text {er }}$ mai 1752, Marc-Pierre de Voyer, comte d'Argenson, écrit à l'Académie Royale des Sciences que le roi a choisi La Galissonière qui avait réuni toutes les voix pour remplacer François Chicoyneau comme associé libre. ${ }^{12}$ Claude de Bonnault a écrit avec inexactitude que Grandjean de Fouchy qui a prononcé l'éloge de Barrin de La Galissonière «ne s'est guère étendu sur les mérites scientifiques du disparu ».13 Grandjean de Fouchy a signalé la contribution de La Galissonière qui a recueilli «partout où il se trouvoit tout ce qui pouvoit fournir matière aux recherches des Naturalistes et des Physiciens. Nos Histoires [les volumes de l'Histoire de l'Académie Royale des Sciences] font mention d'un grand nombre de pièces curieuses qu'il a envoyées à l'Académie et de plusieurs vües qu'il a données sur des articles importants ».14 Grandjean de Fouchy a décrit les grandes lignes de l'activité scientifique de Barrin de La Galissonière, directeur du Dépôt de la Marine.

Dès l'époque de sa formation académique, Roland-Michel Barrin de La Galissonière avait fait preuve de brillantes aptitudes pour les mathématiques. "Déjà très bon mathématicien, a noté l'abbé Rozier, il entra à 17 ans au service en qualité de Garde de la Marine. ${ }^{15} \mathrm{Il}$ a développé le goût de l'étude au Collège de Beauvais, à Paris, sous l'égide du recteur Charles Rollin; «celui-ci charmé de l'application et des talens de son élève prit bientôt pour lui un vif attachement qu'il a conservé jusqu'à sa mort $\gg .^{16}$

Le ministre Jean-Frédéric Phélypeaux de Pontchartrain, comte de Maurepas, avait confié à Duhamel du Monceau la charge 262.

12 Bibliothèque Nationale. Nouvelles Acquisitions françaises, № 9492,

13 Claude de Bonnault, « La Galissonière et sa contribution à la botanique du Canada 》, Les Botanistes français en Amérique du Nord avant 1850. Colloques internationaux du Centre National de la Recherche Scientifique (Paris, 1956), 176.

14 Histoire de l'Académie Royale des Sciences, année 1756, 151.

15 M. l'abbé Rozier, Nouvelle Table des articles contenus dans les volumes de l'Académie Royale des Sciences de Paris depuis 1666 jusqu'en 1770, dans ceux des Arts et Métiers publiés par cette Académie, et dans la Collection Académique (4 vol., Paris, 1775-1776), 4: 129. A l'avenir: Nouvelle Table.

16 Jean-Paul Grandjean de Fouchy, «Eloge de M. le Marquis de La Galissonière », Histoire de l'Académie Royale des Sciences, année 1756, 148. 
d'Inspecteur général de la Marine en compensation du poste d'Intendant du jardin du Roi qui fut accordé à Georges Louis Leclerc de Buffon. ${ }^{17}$ Dans la correspondance échangée avec Duhamel du Monceau, ${ }^{18}$ entre 1739 et 1756 , on se rend compte de la continuité de l'intérêt de Barrin de La Galissonière pour l'histoire naturelle, à partir de notes relatives à la croissance des plantes et des arbres jusqu'aux expériences d'horticulture. ${ }^{19}$ La Galissonière donne suite à ses lectures scientifiques comme l'atteste cette demande au sujet des platanes. «M. Buffon dit dans un de ses mémoires en avoir semé chés luy, vous pouvés savoir comment ils luy ont réussi $\gg,{ }^{20}$ écrit-il à Duhamel du Monceau.

Barrin de La Galissonière fait parvenir au Jardin du Roi ainsi qu'à Duhamel du Monceau des envois contenant parfois, selon son expression, "une assés ample récolte de plantes et de semences $\gg .{ }^{21}$ Voici un extrait de sa lettre du 16 février 1742 qui montre avec quel soin il s'occupait de l'expédition des échantillons de matières botaniques:

J'ai peur que vous ou M. de Jussieu n'aiés laissé passer la première [caisse] sans voir une feuille que je n'y avois mise que pour lui et que je crois estre d'une espèce d'arum des Indes qui est fort beau et dont j'ai une plante que j'envoierai, si l'on veut, au Jardin du Roi, ne l'aiant acceptée qu'à cette intention. ${ }^{22}$

La Galissonière s'enquiert de l'état des travaux horticoles qu'effectue Duhamel du Monceau sur la transplantation des arbres. ${ }^{23}$ A partir de résultats d'expériences, il présente ses vues sur la greffe. ${ }^{24}$ « Je lirai avec grand plaisir, écrit-il à Duhamel du Monceau, votre mémoire sur les marcottes et les boutures

17 Roger Heim, éd., Buffon. Les Grands Naturalistes Français (Paris, 1952), 25.

18 La liste des publications scientifiques de Duhamel du Monceau se trouve dans le répertoire bibliographique de l'abbé Rozier, Nouvelle Table, 4: 157.

19 Archives Publiques du Canada, M. G. 18, G 11.

24.

20 La Galissonière à Duhamel du Monceau, Toulon, 4 avril 1743, Ibid.,

21 Id. à $I d$., $A$ bord du Rubi, 12 décembre 1739, Ibid., 4.

$22 I d$. à $I d$., Toulon, 16 février $1742, I b i d ., 10$.

23 Id. à $I d$., $A$ bord du Rubi, 12 décembre 1739 , Ibid., 5 .

24 Id. à $I d$., Toulon, 14 mars 1743, Ibid., 21. 
et je vous en dirai mon avis avec toute la franchise que vous me connaissés et qui vous est due. ${ }^{25}$

$\mathrm{Au}$ cours de périodes d'absences prolongées, Barrin de La Galissonière dirigeait des expériences botaniques; il adressait à Denis Moreau, horticulteur à son service, des mémoires qui nous renseigneraient sur sa méthode de recherche. Dans une lettre rédigée peu avant sa mort, il s'inquiète de l'état de ses propres expériences de botanique et s'exprime en ces termes:

Je vous félicite de la réussite des sabots de la Vierge et je vous exorte à en mettre auprès quelques uns de l'espèce d'Europe, afin d'avoir des variétés. Je ne sai pas depuis longtemps comme ont fait les miens, non plus que les tulippiers. J'ai pourtant eu, depuis quelques jours, un petit mémoire de Denis Moreau qui me rend compte de bien des choses, mais fort légèrement et il en a oublié encore davantage. Je vous prie de le gronder un peu là-dessus, car je lui envoie sa besogne toute mâchée, c'est à dire des mémoires tous faits où il n'y a, la plus part du temps qu'un oui ou un non à mettre en marge. Il m'a laissé mourir beaucoup de mûriers, faute de culture et beaucoup de pêchers, faute d'arosage dans cet été cy-mesme, qu'on me mande avoir été fort pluvieux en Bretagne. ${ }^{26}$

Les échanges scientifiques avec $M$. de Jussieu, ${ }^{27}$ un des membres de la célèbre famille de botanistes, ne me sont connus que par les indications qui se trouvent dans les lettres adressées à Duhamel du Monceau. Barrin de La Galissonière expédie à Jussieu des échantillons de matières botaniques, ${ }^{28}$ il lui communi-

25 Id. à $I d$., Brest, 14 mai 1744, Ibid., 25.

26 Id. à $I d$., Toulon, $1^{\text {er }}$ septembre 1756, Ibid., 31-32.

27 Le prénom n'est pas indiqué. C'était probablement Bernard de Jussieu qui faisait partie du cercle d'amis de La Galissonière d'après une lettre de Jean-François Gaulthier à Jean-Etienne Guettard, Québec, 21 octobre 1752: «... vous avés occasion de voir souvent Mrs de La Galissonière, Du Hamel et Bernard de Jussieu ....». Bibliothèque centrale du Muséum national d'histoire naturelle, ms. 293, pièce 2 , fol. 2 . Bernard de Jussieu (Lyon, 17 août 1699 - Paris, 6 novembre 1777), médecin et botaniste, il fut membre de l'Académie Royale des Sciences et remplaça Tournefort au Jardin du Roi.

28 La Galissonière à Duhamel du Monceau, à bord du Tigre, en rade de Toulon, 7 octobre 1741, APC. M.G. 18, G 11, 7; Id. ̀̀ Id., Toulon, 16 février 1742, Ibid., 9. 
que une explication relative à un coquillage, ${ }^{29}$ il lui fait parvenir un mémoire intéressant la botanique..$^{30}$

Roland-Michel Barrin de La Galissonière participe à l'activité des chercheurs tels que Jussieu et Guettard qui se rangent parmi les initiateurs de Antoine-Laurent Lavoisier dans le domaine de la recherche scientifique. ${ }^{31}$ Le naturaliste Jean-François Gaulthier, établi au Canada et actif collaborateur de Barrin de La Galissonière, s'enquiert auprès de Jean-Etienne Guettard du succès des échantillons de matières botaniques qu'il a expédiés à René-Antoine Ferchault de Réaumur. ${ }^{32}$ Il lit avec «une vraie satisfaction », selon son expression, le mémoire de Guettard sur les minéraux du Canada. «Je suis charmé, écrit il à Guettard, que le peu de minéraux que je vous ai envoyé, l'année dernière, vous ait fait plaisir. Le bon usage que vous en faites est un motif puissant pour moi, et qui m'engagera à continuer ces sortes d'envoys autant qu'il me sera possible d'en avoir de toutes les parties du Canada. $\gg^{33}$

Jean-François Gaulthier signale la contribution à la fois politique et scientifique de La Galissonière au Canada.

Il est certain que le Canada contient bien des raretés et des choses intéressantes en fait d'histoire naturelle. C'est un pays tout neuf et d'où on n'a encore rien tiré pour ainsi dire parce que nous n'avons presque jamais eû aucun gouverneur ni aucun intendant qui aient voulu s'intéresser à ces sortes de recherches. M. le marquis de La Galissonière est le seul qui ait commencé à mettre les choses sur un bon pied. Le Canada a fait une très grande perte en le perdant. La vaste étendue de ses connaissances jointe à son grand amour pour le bien public et pour tout ce qui peut être utile à l'Etat l'auroit porté à établir solidement une colonie qui est presque naissante ... .34

29 Id. à Id., A bord du Tigre, en rade de Toulon, 7 octobre 1741, Ibid., 7. 30 Id. à Id., Toulon, 2 décembre 1742, Ibid., 12. 63.

31 D. McKie, Antoine Lavoisier, Scientist, Economist, Social Reformer,

32 Jean-François Gaulthier à Jean-Etienne Guettard, Québec, 21 octobre 1752. Bibliothèque centrale du Muséum national d'histoire naturelle, ms. 293, pièce 2 , fol. 1 .

33 Ibid.

34 Ibid., fol. 1-1v. 
Jean-François Gaulthier ajoute que La Galissonière «entroit dans une infinité de détails qui faisaient plaisir aux habitans de cette colonie $\gg^{35}$

Les relations scientifiques de Barrin de La Galissonière et de Louis-Guillaume Le Monnier ${ }^{36}$ sont indiquées dans deux lettres conservées à la Bibliothèque centrale du Muséum national d'histoire naturelle. La Galissonière avait communiqué à Le Monnier un «extrait d'une lettre de l'Orient du 24 octobre $1751 \gg$; il lui demandait d'identifier des échantillons de matières botaniques qu'il lui faisait parvenir: "vous me Ferés un sensible Plaisir et vous rendrés peut être quelque service à la botanique en faisant la dessus les recherches que vous pourrés puisque cela engagera la personne de qui nous tenons ces graines cy a nous en procurer d'autres. ${ }^{37}$

Quels furent les rapports scientifiques de La Galissonière avec les naturalistes des pays étrangers? Les pièces qu'il me fut possible d'inventorier dans la correspondance échangée entre La Galissonière et les représentants britanniques ne portent que sur des sujets militaires et politiques. Au début de juillet 1749, La Galissonière avait accueilli le botaniste suédois, Pehr Kalm, disciple de Linné. ${ }^{38}$ L'année précédente, Kalm avait rendu visite à Hans Sloane; ${ }^{39}$ avant d'arriver au Canada, il avait établi des relations fructueuses dans les colonies britanniques avec Benjamin Franklin et John Bartram. D'après des documents conservés à la bibliothèque de l'American Philosophical Society, Gilbert Chinard a noté qu'en botanique Franklin a joué son rôle accoutumé d'intermédiaire entre les savants français et britanniques. ${ }^{40}$

\section{Ibid.}

36 Louis-Guillaume Le Monnier (Paris, 27 juin 1717 - Montreuil, 7 septembre 1799). Elève de Bernard de Jussieu, il devint professeur de botanique au Jardin du Roi.

$37 \mathrm{La}$ Galissonière à Le Monnier, Fontainebleau, 5 novembre 1751. Bibliothèque centrale du Muséum national d'histoire naturelle. 107.

38 W. O. James, «Linné, 1707-1778», Endeavour, XVI, no 62 (1957),

39 G. R. de Beer, Sir Hans Sloane and the British Museum (Londres, 1953), 137, 156; Basil Williams, The Whig Supremacy, 1714-1760 (Londres, 1952), 363.

40 Les Botanistes français en Amérique du Nord avant 1850, 284. 
Dans un article intitulé: "Linné, Kalm et l'étude de la flore nord-américaine au XVIII ${ }^{e}$ siècle ${ }^{41}$ C. Skottsberg a décrit les principales étapes et les résultats du voyage scientifique de Kalm en Amérique. Pehr Kalm a fait allusion à l'étendue et à la variété des connaissances scientifiques de La Galissonière qui lui rappelait son maître Linné. ${ }^{2}$

Le $\mathrm{P}$. Bonnécamps, S.J. représente la tradition scientifique des Jésuites en Nouvelle-France. A la demande de La Galissonière, il a accompagné l'expédition de Céloron de Blainville à la Belle Rivière [Ohio], en 1749. Sa relation de voyage contient des renseignements d'ordre botanique, hydrographique et géographique. ${ }^{43}$ «La nécessité d'avoir de pareilles connaissances, écrit La Galissonière au ministre Rouillé, m'a engagé à envoyer au détroit le $\mathrm{Sr}$ de Léry fils et à Missilimakinak le $\mathrm{Sr}$ de Lotbinière. Ils n'ont point d'autre mission que d'observer tout ce qui peut être utile au service et d'en dresser des mémoires. Le P. Bonecamp a fait faire pour le Sr de Léry et pour lui des instruments pour prendre [la] hauteur et les a divisés, mais le $\mathrm{Sr}$ de Lotbinière a gradué le sien lui même et $\mathrm{j}$ 'ai beaucoup de confiance à son travail. ${ }^{44}$

Barrin de La Galissonière a communiqué avec les astronomes et les physiciens de son temps à propos d'astronomie nautique et d'hydrographie. "Si je puis tirer de M. Bouguer une réponse à vos remarques sur l'octant de M. Hadley je vous la ferai aussi passer $\gg,{ }^{45}$ écrit-il à Chartier de Lotbinière. Il a

41 Ibid., 182.

42 Pehr Kalm, Travels into North America (Traduction de John Reinhold Forster. 2 vol., Londres, 1772), 2: 369.

43 R. G. Thwaites, éd., « Relation de voyage de la Belle Rivière fait en 1749 sous les ordres de M. de Céloron par le P. Bonnécamps », The Jesuit Relations and Allied Documents, 69: 150-199; Auguste Gosselin, «Le Père de Bonécamps, dernier professeur d'hydrographie au Collège de Québec, avant la conquête 》, Mémoires et Comptes rendus de la Société Royale du Canada, seconde série, tome 1 (1895), 38; L. H. Gipson, Zones of International Friction: North America, South of the Great Lakes Region, 1748-1754. The British Empire before the American Revolution (New York, 1939), $4: 170,196$.

44 Québec, le 26 juin 1749. APC. Transcription tirée des Archives des Colonies, série C11A, $93: 141$.

45 La Galissonière à Lotbinière, Paris, 17 mars 1755. Archives Publiques du Canada. Papiers de Lotbinière, No 27-105, 2, 1753-1759, M. G. 18, $\mathrm{K} 3,2$ (2). 
rédigé des mémoires scientifiques ${ }^{46}$ en collaboration avec les astronomes Pierre Bouguer ${ }^{47}$ et Pierre-Charles Le Monnier ${ }^{48}$ et le géographe Philippe Buache. ${ }^{49}$

L'abbé Rozier a résumé l'œuvre accomplie par La Galissonière au «Dépôt des plans, cartes et Journaux de la Marine: il falloit en rectifier plusieurs [cartes] par des observations astronomiques, et $M$. de La Galissonière se chargea de les faire exécuter; il enrichit singulièrement ce Dépôt en y ajoutant la nombreuse collection de M. de Lisle.$_{.50} \mathrm{Au}$ cours de sa vie studieuse, La Galissonière a été dirigé par des préoccupations scientifiques; "son goût pour l'histoire naturelle le suivit par-tout, et il sut lui donner tous les instans de liberté que lui laissoit le service du Roi ». ${ }^{51}$

Les rapports qui ont existé entre Roland-Michel Barrin de La Galissonière et les hommes de sciences de la première moitié du XVIII ${ }^{e}$ siècle illustrent la participation de celui qui fut gouverneur intérimaire de la Nouvelle-France à l'œuvre de collaboration nécessaire à l'accumulation d'observations rigoureuses dans les domaines des sciences naturelles, de l'astronomie nautique et de l'hydrographie.

Roland Lamontagne, Ph.D., Faculté des Arts, Université de Montréal.

46 Voici les titres: «Le 6 septembre 1752. - Rapport de Bouguer, Le Monnier et La Galissonière sur un ouvrage de Chabert et voyage sur les côtes de l'Amérique septentrionale pour déterminer les longitudes géographiques. Le $1^{\text {er }}$ décembre 1753. - Rapport de La Galissonière, Bouguer, Le Monnier et Buache sur les nouvelles cartes de M. D'Après. Le 13 mars 1756. - Rapport de La Galissonière et Le Monnier sur des observations hydrographiques faites sur les côtes d'Espagne par M. Chabert ». Bibliothèque Nationale. Nouvelles acquisitions françaises, No 9492, 262.

47 Nouvelle Table, 4: 41.

$48 \mathrm{M}$. Daumas, Les Instruments scientifiques au XVII et XVIIIe siècles (Paris, 1953), 163.

49 Nouvelle Table, $4: 53$.

so Ibid., $4: 129$.

51 Ibid. 